\title{
Characterization of Starch Synthetic Genes and Starch Granule during Seeds Development between Synthetic Hexaploid Wheat and its Parents
}

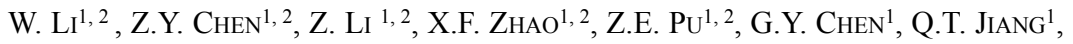 \\ Y.M. WeI ${ }^{1}$ and Y.L. ZHENG ${ }^{1 *}$ \\ ${ }^{1}$ Triticeae Research Institute, Sichuan Agricultural University, Chengdu, Sichuan 611130, China \\ ${ }^{2}$ College of Agronomy, Sichuan Agricultural University, Chengdu, Sichuan 611130, China
}

(Received 18 September 2016; Accepted 6 November 2017;

Communicated by R.N. Chibbar)

\begin{abstract}
To study the development of starch granules in polyploid wheats, we investigated the expression of starch synthetic genes between the synthetic hexaploid wheat SHW-L1, its parents T. turgidum AS2255 and diploid Ae. tauschii AS60. The synthetic hexaploid wheat SHW-L1 showed significantly higher starch content and grain weight than its parents. Scanning electron microscopy (SEM) showed that SHW-L1 rapidly developed starch granules than AS2255 and AS60. The amount of B-type granule in AS60 was less than that in SHW-L1 and AS2255. RT-qPCR result showed that the starch synthetic genes AGPLSU1, AGPLSU2, AGPSSU1, AGPSSU2, GBSSI, SSIII, PHO1 and PHO2 expressed at earlier stages with larger quantity in SHW-L1 than in its parents during wheat grain development. The expression of the above mentioned genes in AS60 was slower than in SHW-L1 and AS2255. The expression pattern of starch synthase genes was also associated with the grain weight and starch content in all three genotypes. The results suggested that the synthetic hexaploid wheat inherited the pattern of starch granule development and starch synthase gene expression from tetraploid parent. The results suggest that tetraploid wheat could plays more important role for starch quality improvement in hexaploid wheat.
\end{abstract}

Keyword: starch synthase, starch granule, seed, synthetic hexaploid wheat, tetraploid

\section{Introduction}

Grain yield is largely determined by starch accumulation during the grain filling phase in cereals. Wheat grains mainly consist of starch (60-80\%) and proteins (10-15\%), the remains include cellulose, fat, and mineral substances (Smith et al. 1995). Starch of the wheat endosperm consists of amylose (20-30\%) and amylopectin (70-80\%) (Hurkman et al. 2003; Sestili et al. 2010). Amylose is synthesized by ADP glucose pyrophosphorylase (AGPase) and granule-bound starch synthase (GBSS), while amylopectin is synthesized by the AGPase, starch synthases (SS), starch branching enzyme ( $S B E)$, and starch debranching enzyme $(D B E)$. Disproportionating enzyme $(D P E)$ and phosphorylase $(P H O)$ 
are considered to be involved in starch biosynthesis and degradation (Colleoni et al. 1999; Ball and Morell 2003; Tetlow et al. 2004). GBSS is essential for amylose synthesis and exclusively bound to the starch granule. SSI, SSII, SSIII, and SSIV are responsible for amylopectin chain elongation with their distribution between the granular and soluble fractions (Ball and Morell 2003; Li et al. 2003). Two types of starch granules, type A granules $(>15 \mu \mathrm{m})$ and type $\mathrm{B}$ granules $(<15 \mu \mathrm{m})$, exist in wheat endosperm. The content and ratio of amylose and amylopectin, as well as the granule type and ratio, strongly affect grain yield and the end product utilization of wheat. New germplasm resource with diverse starch properties is very valuable for wheat improvement. Therefore, identification of new alleles of starch synthetic genes can help to improve wheat grain quality. Recently, some alleles of starch synthetic genes have been identified in Triticum and Aegilops species. Two types of waxy gene in T. urartu and Ae. tauschii, respectively (Murai et al. 1999), alleles $W x-A m 1^{a}$ and $W x-A m 1^{a}$ in Spanish T. monococcum (Guzmánet al. 2009), null SGP-A1 alleles (gene SSIIa) (Hogg et al. 2013) and alleles $W x-B 1 c$ ' and $W x$ $B 1 f$ in durum wheat (Nieto-Taladriz et al. 2000), were observed. The variation of SNPs (single nucleotide polymorphisms) and InDels (insertions or deletions) of waxy gene produced new alleles in Triticum and Aegilops (Li et al. 2012; Yamamori and Guzman 2013). The significant differences of $W x-A 1$ and $W x-B 1$ between diploid and hexaploid wheat also have been reported (Huang and Brûlé-Babel 2010). Phylogenetic analysis suggested that $W x-A^{u} 1$ and $W x-A^{m} 1$ of diploid wheat were clearly different from the $W x-A 1$ loci of tetraploid and hexaploid wheat ( $\mathrm{Li}$ et al. 2015). These findings suggested that the starch synthetic genes had diversified variation in Triticum and Aegilops species. These alleles have potential useful value for starch improvement of wheat breeding.

Synthetic hexaploid wheat provides a good strategy to evaluate the change of genes during polyploidization in wheat. The differences of protein composition and quality between synthetic wheats and their parents have been reported (Pena et al. 1995). It has been suggested that the tetraploid parents often exert greater influence on the properties of the synthetic hexaploid than diploid parents (Gororoet al. 2001). The little difference in the amylose contents and amylose-lipid dissociation peak temperatures between synthetic hexaploid wheat and lower ploidy wheats was also observed (Konik-Rose et al. 2009). However, there are only a few reports on the starch properties of synthetic hexaploid wheat and their parents (Gororoet al. 2001; Konik-Rose et al. 2009).

The aim of this study was to evaluate the performance of starch granule development and the expression of starch synthetic genes in synthetic hexaploid wheat and its parents.

\section{Materials and Methods}

\section{Plant materials}

Synthetic hexaploid wheat SHW-L1 $(2 \mathrm{n}=6 \mathrm{x}=42$, AABBDD), and its parents, $T$. turgidum landraces AS2255 $(2 \mathrm{n}=4 \mathrm{x}=28, \mathrm{AABB})$ and Ae.tauschii AS60 ( $2 \mathrm{n}=2 \mathrm{x}=14$, DD), used in this study were provided by Triticeae Research Institute, Sichuan Agricultural University (Zhang et al. 2004). All the plants were grown during 2014-2015 in a field 
(longitude 103.88 E, latitude 30.73 N) of Sichuan Agricultural University, Chengdu city, Sichuan Province, China.

\section{Determination of grain weight and starch concentration}

The mature seeds were collected from all the plants. The 1000-grain weight was determined using an electric balance (BS200S-WE1, Sartorius, Gottingen, Germany) in three replications for each accession. The starch content was determined in triplicate using total starch assay kit (Megazyme, Ireland). Mature seeds were cut into halves and stained in $0.1 \%(\mathrm{w} / \mathrm{v})$ iodine solution $(1.0 \mathrm{~g}$ of potassium iodide and $0.1 \mathrm{~g}$ of iodine diluted to 100 $\mathrm{mL}$ with distilled water). Amylose content of samples was measured by the dual-wavelength colorimetric method (Zhu et al. 2008). Amylopectin content was calculated through starch content minus amylose content. Data was analyzed by ANOVA and the means were compared by the least significant difference (LSD) test.

\section{Scanning electron microscopy (SEM) analysis}

During the flowering period, the pollinating spikelets were tagged in SHW-L1, AS2255, and AS60, respectively. The kernels in the middle of spike were collected at 3, 6, 9, 12, $15,18,21,24,27$, and 30 DAP (days after pollinated), respectively. These kernels were transversely sectioned across the centre by knife and mounted on circular aluminum specimen holders with double-sided sticky tape. The newly broken surface of kernels was directly viewed and photographed with a FEI Quanta 450 Environmental SEM (FEI Company, Hillsboro, Oregon State, USA).

\section{RNA extraction, $c D N A$ synthesis and real-time quantitative PCR (RT-qPCR)}

The kernels of the spikelets located on the middle spike of SHW-L1, AS2255, and AS60 were collected at $6,12,18,24$, and 30 DAP, respectively. Total RNAs were extracted from these kernels with RNAprep pure Plant Extraction Kit (DP432) (Tiangen Biotech Co. Ltd, Beijing, China). The cDNA was synthesized from reverse transcription using a PrimeScript ${ }^{\mathrm{TM}} 1^{\text {st }}$ Strand cDNA Synthesis Kit (Takara, Dalian, China). The primers of RT-qPCR (Table 1) were designed based on the sequences DQ839506 (AGPLSU1), DQ406820 (AGPLSU2), EF405961 (AGPSSU), AY050174 (GBSSI), AF258608 (SSIII), EU595762 (PHO1), AF275551 (PHO2) from GenBank (http://www. ncbi. nlm. nih. gov). The PCR products were sequenced to confirm the amplification (Sangon Biotech Co., Ltd. Shanghai, China). Each PCR reaction in a total volume of $20 \mu$ contained $9 \mu \mathrm{l} \mathrm{SYBR}$ Green Master Mix (Tiangen Biotech Co. Ltd., Beijing, China), $1 \mu$ l of cDNA and $100 \mathrm{nM}$ of both forward and reverse primers. The RT-qPCRs were performed in the BIO-RAD MyiQ Real Time PCR (BIO-RAD, Hercules, California, USA). The data were analyzed via the Bio-Rad iQ5 software. The ACTIN gene (AB181991) was used as an internal reference and control for expression analysis. The RT-qPCR program included an initial denaturation at $95^{\circ} \mathrm{C}$ for $2 \mathrm{~min}$, followed by 40 cycles of $95^{\circ} \mathrm{C}$ for $5 \mathrm{~s}, 60{ }^{\circ} \mathrm{C}$ for $15 \mathrm{~s}$, 
Table 1. The primers of starch synthetic genes used in RT-qPCR

\begin{tabular}{|c|c|c|c|c|c|c|}
\hline Gene name & $\begin{array}{c}\text { NCBI } \\
\text { number }\end{array}$ & $\begin{array}{l}\text { Name of } \\
\text { primer }\end{array}$ & The sequence of primer $\left(5^{\prime}-3^{\prime}\right)$ & $\begin{array}{c}\text { Amplified } \\
\text { region (bp) }\end{array}$ & $\begin{array}{l}\text { Product of } \\
\text { PCR (bp) }\end{array}$ & $\begin{array}{l}\mathrm{Tm} \\
\left({ }^{\circ} \mathrm{C}\right)\end{array}$ \\
\hline \multirow[t]{2}{*}{$A G P L S U 1$} & DQ839506 & $\mathrm{F} 1$ & CACATTCACCGCACCTAC & $573-720$ & 148 & 52.8 \\
\hline & & $\mathrm{R} 1$ & CCTCAAGCACCCAGATAA & & & \\
\hline \multirow[t]{2}{*}{$A G P L S U 2$} & DQ406820 & $\mathrm{F} 2$ & CATCACGCAGAAACCTACC & 1094-1217 & 124 & 49.8 \\
\hline & & $\mathrm{R} 2$ & GAGAACGGATTCCAACGA & & & \\
\hline \multirow[t]{2}{*}{$A G P S S U$} & EF405961 & F3 & TACGATGGTTACTGGGAAGAT & $887-1030$ & 144 & 49.2 \\
\hline & & R3 & TGAAGGAGGCAAGTGTCG & & & \\
\hline \multirow[t]{2}{*}{ GBSSI } & AY050174 & $\mathrm{F} 4$ & CCTCAACAACAACCCACA & $766-913$ & 148 & 53.7 \\
\hline & & R4 & ATTGGACTGGTAGTTGCTCT & & & \\
\hline \multirow[t]{2}{*}{ SSIII } & AF258608 & F5 & ACGGTCGCACGGTCTATGAG & $2460-2638$ & 178 & 52.3 \\
\hline & & R5 & CTTTCTTCGCCGCTGTTCTT & & & \\
\hline \multirow[t]{2}{*}{$\mathrm{PHOl}$} & EU595762 & F6 & GATGTTCCTATTCCTGGCTAC & $912-1097$ & 186 & 50 \\
\hline & & R6 & CTCTGATGATTCGTCCCCT & & & \\
\hline \multirow[t]{2}{*}{$\mathrm{PHO2}$} & AF275551 & F7 & CAGAGATGGAACGACACCT & $232-353$ & 152 & 56.5 \\
\hline & & R7 & AACTTCTTCAGGGCGTCA & & & \\
\hline \multirow[t]{2}{*}{$A C T I N$} & AB181991 & $\mathrm{F}$ & TACTCCCTCACAACAACCG & $367-508$ & 142 & 52.3 \\
\hline & & $\mathrm{R}$ & CCATCAGGCATCTCATAGC & & & \\
\hline
\end{tabular}

and $72{ }^{\circ} \mathrm{C}$ for $15 \mathrm{~s}$, and a melt curve stage. Relative expression levels of all genes were calculated using the Pfaffl method (Pfaffl 2001).

\section{Results}

\section{Thousand-grain weight and starch content}

The average 1000-grain weight in AS2255, SHW-L1 and AS60, were $25.63 \mathrm{~g}, 24.35 \mathrm{~g}$ and $5.42 \mathrm{~g}$, respectively. AS2255 had significantly higher 1000-grain weight than SHWL1, while SHW-L1 had significantly higher value than AS60 by Duncan multiple comparisons. SHW-L1 (52.7\%) showed slightly higher value of starch content than that of AS2255 (51.8\%) and AS60 (44.7\%). The amylose content of SHW-L1 (15.6\%) was higher than that of AS2255 (14.7\%) and AS60 (12.8\%), whereas the amylopectin content of SHW-L1 (37.0\%) and AS2255 (37.1\%) were significantly higher than that of AS60 $(31.9 \%)$. These results show that the synthetic hexaploid wheat SHW-L1 had similar performance of grain weight and starch content to the tetraploid parent. 


\section{Development of starch granules}

Scanning electron microscopic (SEM) analysis revealed that at three DAP, most of A type starch granules $(>10 \mu \mathrm{m})$ enriched in the amyloplast were observed in all the three genotypes (Fig. 1). The producing of A type starch granules from the amyloplast was observed in AS2255. At six DAP, the membrane of amyloplast was disappearing and most of

A
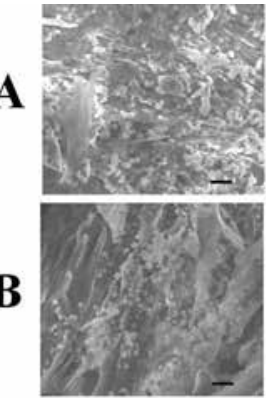

C

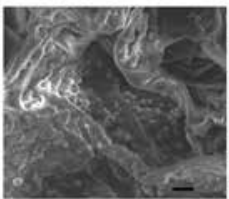

a
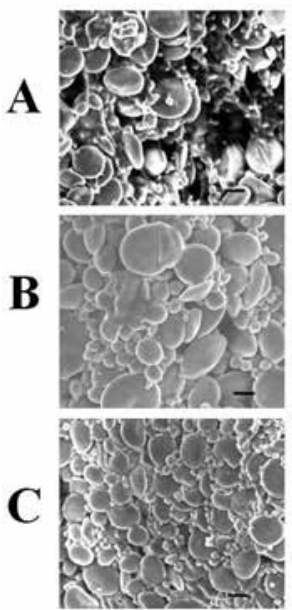

f
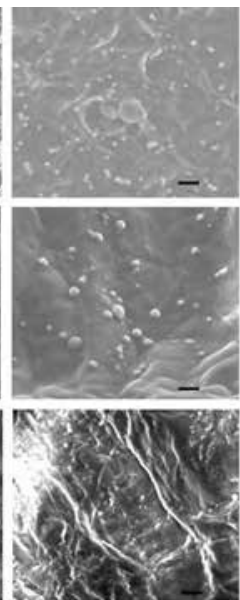

b
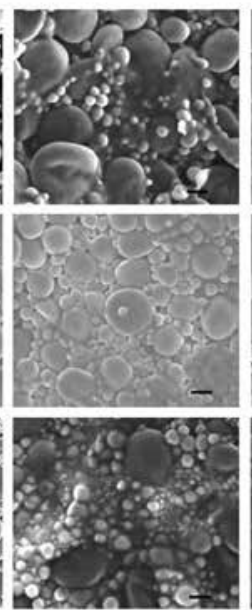

g

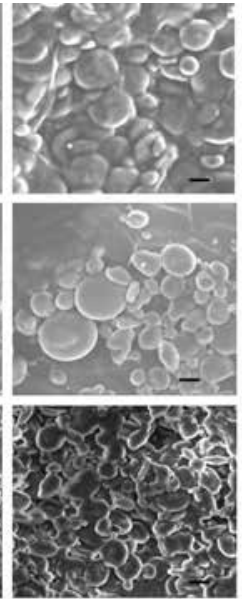

c
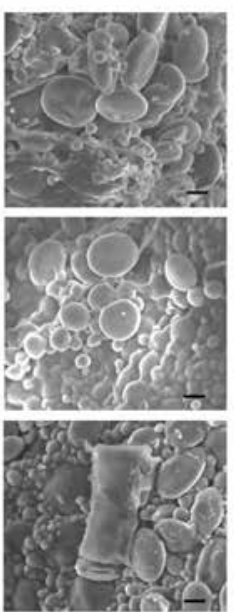

h

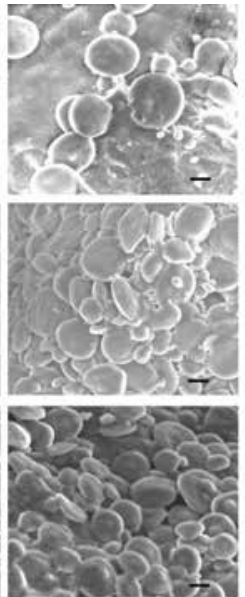

d
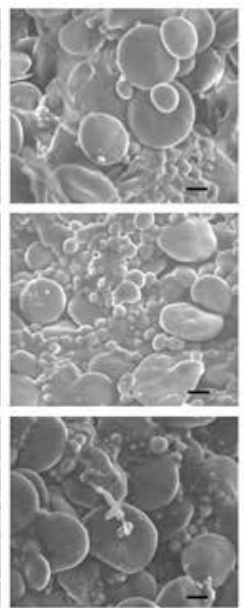

i

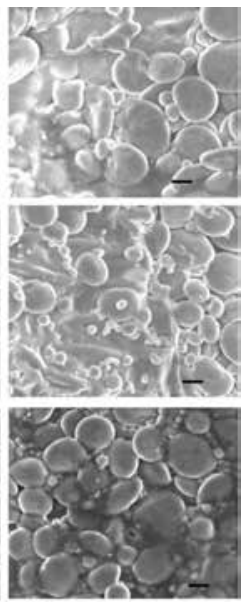

e
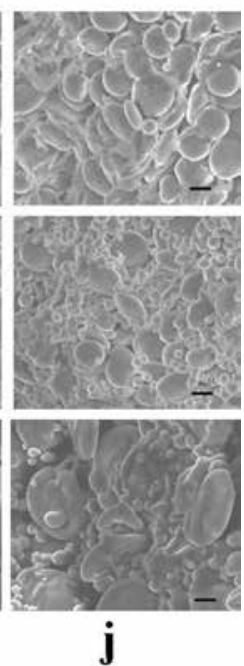

Figure 1. Starch granule of diploid Ae. tauschii AS60(A), tetraploid T. turgidum AS2255(B), and synthetic hexaploid wheat SHW-L1(C) visualized by SEM. These images of transverse grain sections represent 10 developmental stages, including at 3 DAP (a), 6 DAP (b), 9 DAP (c), 12 DAP (d), 15 DAP (e), 18 DAP (f), 21 DAP (g), 24 DAP (h), 27 DAP (i), and 30 DAP (j), respectively. For each accession and stage, at least three seeds were examined and similar results were observed. Magnification is $\times 3000$. Scale bars represent $10 \mu \mathrm{m}$ 
A-type granules were dissociating from the amyloplasts. AS2255 and SHW-L1 showed faster dissociating process than AS60. At nine DAP, both of A-type and B-type starch granules were observed. AS2255 and SHW-L1 showed faster appearance of granules than AS60. At 12 DAP, the B-type starch granules were observed in all accessions. At 15 DAP, the amount of B-type granules had been increased rapidly in all the three genotypes, but AS2255 and SHW-L1 showed larger number of B-type granules than AS60. At 18 DAP, the B-type granules were observed clearly and distributed evenly in AS2255 and SHWL1. At 21 DAP, the amount of B type granule had the first peak value, and the smaller size $(<5 \mu \mathrm{m}) \mathrm{C}$-type starch granule appeared. At $30 \mathrm{DAP}$, the quantity of A type granule did not change and reached a stable stage. Although the amount of B type granules had increased in three genotypes compared with the last stage, the less quantity of B type granules in AS60 was observed compared with AS2255 and SHW-L1.

\section{Expression patterns of starch synthase genes}

DNA sequencing confirmed that the fragments amplified with the designed primers were the expected genes (Table 1). All primers can be used to detect the expression of these genes in RT-qPCR. The expression of AGPLSU1, AGPLSU2, AGPSSU, GBSSI, SSIII, $P H O 1$, and $P H O 2$ showed significant differences between the synthetic hexaploid wheat SHW-L1 and the tetraploid wheat AS2255 and diploid AS60 at five developing stages of seed by RT-qPCR (Fig. 2). At six DAP, the expression of five genes, except AGPLSU2 and GBSSI in AS60, were detected in all three accessions. AGPLSU1 and AGPSSU had the highest expression in AS60 than in AS2255 and SHW-L1. GBSSI and PHO1 had the highest expression in AS2255, followed by SHW-L1 and AS60. SSIII and PHO2 had the highest expression in SHW-L1, followed by AS60 and AS2255. AGPLSU2 had higher expression in SHW-L1 than in AS2255. AGPLSU2 and GBSSI had higher expression level than the other genes at this stage. At 12 DAP, the expression of $A G P L S U 1$ and $A G-$ PLSU2 in SHW-L1, and AGPLSU2 and GBSS1 in AS60, were not detected. The expression of AGPLSU1, SSIII, and PHO2 had the highest level in AS60, followed by AS2255 and SHW-L1. AGPLSU2 and GBSSI had the highest expression in AS2255. PHO1 had the highest expression in SHW-L1, implied that the degradation of starch had been activated in SHW-L1. The expression of gene AGPSSU had insignificant difference among AS60, AS2255, SHW-L1. At 18 DAP, AGPLSU1 had the highest expression in AS2255 than in AS60 and SHW-L1. AGPLSU2, SSIII, PHO1, and PHO2 had the highest expression level in AS60, compared with AS2255 and SHW-L1. The expression of GBSSI was not detected in AS60. Otherwise, AGPSSU and GBSSI had the highest expression in SHW-L1, and followed by AS2255 and AS60. At 24 DAP, the expression of AGPLSU1, $A G P L S U 2$, and GBSS1 were not detected in AS60. SHW-L1 showed very low level expression of $A G P L S U 1$, but significantly higher expression level of $A G P S S U$ and GBSS1 than in AS2255 and AS60. At 30 DAP, only the expression of AGPLSU1 was not observed in AS2255. AGPLSU1, AGPLSU2, PHO1, and PHO2, had the highest expression in AS60. AGPSSU2 and GBSSI had higher expression in AS2255 and SHW-L1 than that in AS60. SSIII had the highest expressing level in AS2255 than that in AS60 and 


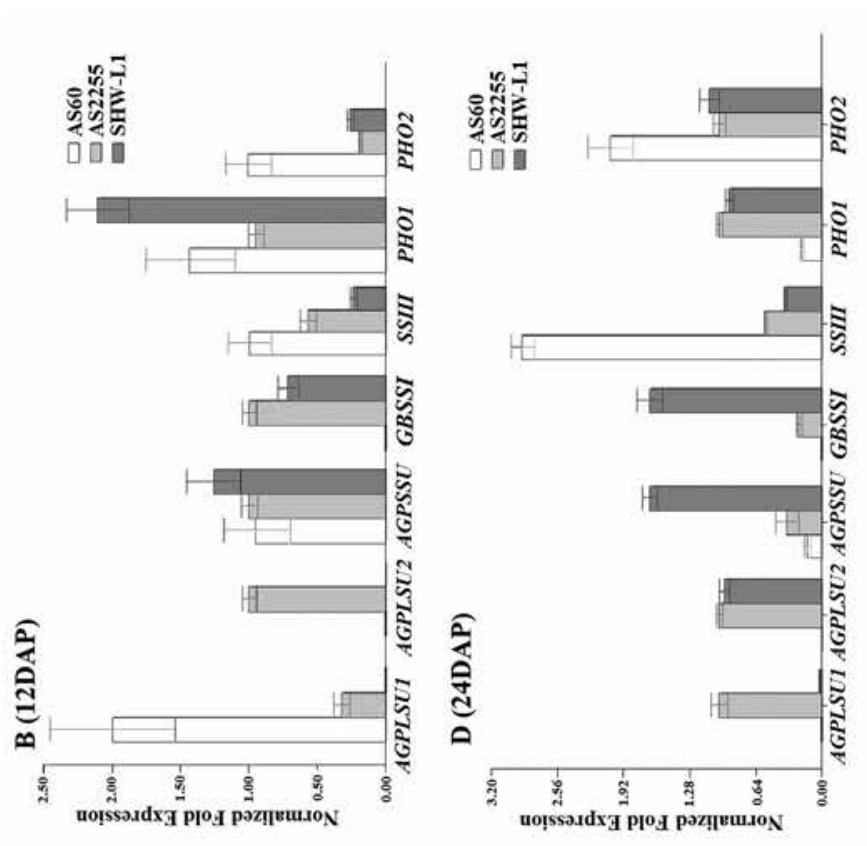

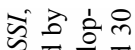

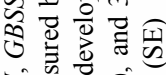

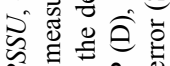
\&

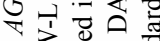
กิ่

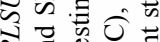
ते $\overrightarrow{0}$ के पि

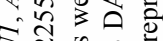
ड़ ते घै

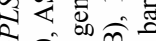
डิ

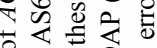

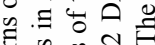
ह i 0

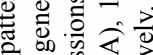
0 , on $\mathbb{E}$

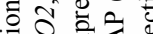

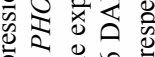
문 爻ठ் ن 넌

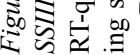

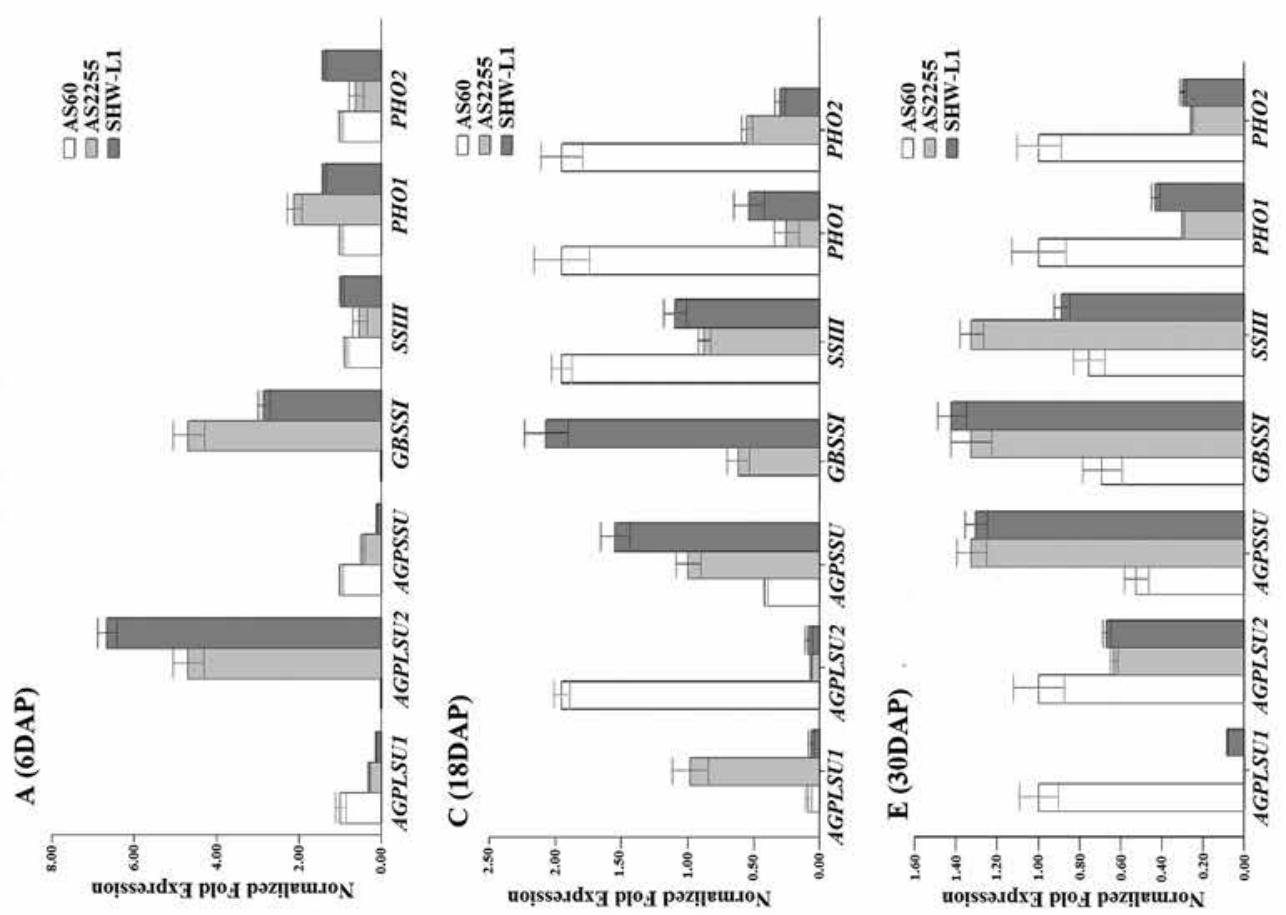

Cereal Research Communications 46, 2018 
SHW-L1. These results showed that most of starch synthase genes had higher and earlier expression in synthetic hexaploid and tetraploid wheat. But PHO1 and PHO2 had been keeping higher activity at the whole stages of seed development in diploid AS60.

\section{Discussion}

The utilization of novel alleles of starch synthase genes in germplasm resources is very valuable for wheat breeding (Konik-Rose et al. 2009; Guzmán et al. 2011). These alleles could strongly affect the characterizations of starch for use in both food and non-food industries (Martin and Smith 1995). A total of 12 accessions with amylose contents above $40 \%$ were reported in T. durum and Ae. tauschii (Watanabe et al. 1998). The previous research showed that the synthetic hexaploid wheats had intermediate swelling power values, and tetraploid durums had the highest swelling powers, and the diploid Ae. tauschii had the highest gelatinisation peak temperatures ( Konik-Rose et al. 2009). In the present results, the synthetic hexaploid wheat SHW-L1 had higher grain weight and starch content, higher amount of B-type granules, and stronger expression of starch synthetic genes than its tetraploid and diploid parents. As wheat grains mainly consist of starch (60-80\%) (Smith et al. 1995), the change of starch content might be one of reasons for the increasing of seed production of synthetic hexaploid wheat.

In wheat, the large granules appear at about 4-7 DAA (days after anthesis) and continue to increase in size throughout the grain filling stage, while small granules were initiated at about 12-14 DAA and remain considerably small at final maturity (Bechtel and Wilson 2003). The development of granules was well consistent with starch synthase activities and relative gene expression (Zhang et al. 2010). The durum wheat had higher $\mathrm{B}$ granule contents than the Ae. tauschii accessions, but not as high as the synthetics (Konik-Rose et al. 2009). In the present result, at three DAP (Figure 1a), most of A- type starch granule enriched the amyloplast, and at nine DAP (Figure 1c), the small granule appeared among three accessions. The granules in Ae. tauschii AS60 developed slowly and showed less quantity compared with the synthetic hexaploid and tetraploid wheat. These results suggested that the development of starch granules is species specific. For the developing pattern and the quantity of granules, hexaploid SHW-L1 were more similar to tetraploid AS2255, suggesting that synthetic hexaploid wheat could be inherited more genetic characterizations of starch from tetraploid wheat than diploid. The similar performance that the tetraploid parents had greater influence on the traits of the synthetic hexaploid than the diploid parent had been reported (Flood et al. 1992; Limin et al. 1995; Gororo et al. 2001).

The small and large subunits of ADP glucose pyrophosphorylase (AGPase SSU and $L S U$ ) were the rate-limiting enzyme of starch synthesis in cereal endosperm (Tomlinson and Denyer 2003). AGPase activities in wheat grain are mainly relative to the activities of cytosolic SSUI (Kang et al. 2010). AGPase LSU II showed the highest expression levels from 6 to $11 \mathrm{DPA}$, and was then down-regulated to trace levels at grain desiccation (Guo et al. 2012). In the present results, the expression of AGPase LSU and SSU in all materials showed the similar developing trend compared to the previous researches, while 
the expression of these genes in AS60 lagged behind in SHW-L1 and AS2255. AGPLSU1 and $A G P S S U$ had the highest expression in AS60 at 6 DAP, implied that the expression of AGPase in synthetic hexaploid wheat and T. turgidum could be activated early.

$G B S S$ and $S S$ are responsible for the synthesis of amylose and amylopectin, respectively (Ball and Morell 2003; Li et al. 2003). GBSSI transcripts were low at the beginning, and dramatically increased at 5 DAF (days after florescence) and reached peaks at 24 DAF and then remained abundantly expressed until 32 DAF at the end of endosperm development (Zhang et al. 2010). In the present study, at most of developing stages, SHW-L1 showed higher expression of GBSSI than AS2255 and AS60, suggesting that the synthetic hexaploid wheat had more activity of GBSSI compared with its parents. The expression of GBSSI in diploid AS60 was only detected at 30 DAP, implied that GBSSI could be lagged and had weak expression and short duration in diploid Ae.tauschii.

SSIIIa produces longer length chains extending between clusters in cereal endosperm (James et al. 2003) and also influences indirectly the final molecular architecture of the starch (Gao et al. 1998). The suppression of SSIII activity reduced amylopectin synthesis and the long chains length of the amylopectin in rice (Dian et al. 2005). The SSIII expressed very high at four DAF (days after florescence) in the superior grains while eight DAF in the inferior grains, and then rapidly reached peaks between 12 and 16 DAF and gradually decreased thereafter (Zhang et al. 2010). In the present result, SSSIII had higher expression at six DAP in AS2255 and SHW-L1, and showed the similar expressing pattern with the previous research. But diploid Ae. tauschii AS60 lagged the expression of SSIII at 12 DAP compared with synthetic hexaploid and T. turgidum.

Plant $\mathrm{PHO}$, including plastidial $\mathrm{PHO}$ and cytosolic $\mathrm{PHO}$, was involved in the process of starch degradation and starch biosynthesis in seeds (Colleoni et al. 1999; Critchley et al. 2001; Satoh et al. 2008). PHO1 transcripts were numerous at the onset of seed development, rapidly increased to peak at $5 \mathrm{DAF}$, and diminished to a low but significant level until 15 DAF. The transcripts of $\mathrm{PHO} 2$ were scarce from the start and dwindled from 5 DAF (Tickle et al. 2009). In our results, the expression of PHO1 and PHO2 in Ae. tauschii AS60 also lagged behind those in synthetic hexaploid and T. turgidum. But PHO2 showed the highest expression in AS60 at 12 DAP. The results suggested that $P H O$ genes were activated in the early developing stages of seed, and the synthase and degradation of starch might be activated at the same time during seed development.

Hexaploid wheat $T$. aestivum (AABBDD) was obtained by the hybridization between a tetraploid T. turgidum (AABB) and a diploid Ae. tauschii (DD) (Huang et al. 2002). The new synthetic hexaploid wheat provides a unique system for the investigation of the polyploidization process. A series of studies have demonstrated that allopolyploid formation or speciation in Triticum and Aegilops species could induce directional sequence elimination, random structural changes, epigenetic modifications, DNA methylation, and gene silencing in gene expression (Liu et al. 1998; Ozkan et al. 2001). Our present results indicated that the synthetic hexaploid wheat had a different expression pattern of starch synthetic genes compared with its tetraploid and diploid parents. The similar results for other traits were also reported (Flood et al. 1992; Gororo et al. 2001; Konik-Rose et al. 2009; Guzmán et al. 2011). Synthetic hexaploid wheat could be used for wheat starch 
improvement. Furthermore, the pattern of gene expression of starch synthases in the synthetic hexaploid SHW-L1 was mainly similar to T. turgidum AS2255, suggested that tetraploid wheat could be play a more important role in the improvement of the starch quality of hexaploid wheat.

\section{Acknowledgments}

This work was supported by The Key Project of National Natural Science Foundation of China (No. 31230053) and The International Science \& Technology Cooperation Program of China (No. 2015DFA30600).

\section{References}

Ball, S.G., Morell, M.K. 2003. From bacterial glycogentostarch: Understanding the biogenesis of the plant starch granule. Annu. Rev. Plant Biol. 54:207-233.

Bechtel, D.B., Wilson, J.D. 2003. Amyloplast formation and starch granule development in hard red winter wheat. Cereal Chem. 80:175-183.

Burton, R.A., Johnson, P.E., Beckles, D.M., Fincher, G.B., Jenner, H.L., Naldrett, M.J., Denyer, K. 2002. Characterization of the genes encoding the cytosolic and plastidial forms of ADP-glucose pyrophosphorylase in wheat endosperm. Plant Physiol. 130:1464-1475.

Colleoni, C., Dauvillée, D., Mouille, G., Buléon, A., Gallant, D., Bouchet, B., Morell, M., Samuel, M., Delrue B., d'Hulst, C., Bliard, C., Nuzillard, J., Ball, S. 1999. Genetic and biochemical evidence for the involvement of a-1,4 glucanotransferases in amylopectin synthesis. Plant Physiol. 120:993-1003.

Critchley, J.H., Zeeman, S.C., Takaha, T., Smith, A.M., Smith, S.M. 2001. A critical role for disproportionating enzyme in starch breakdown is revealed by a knock-out mutant in Arabidopsis. Plant J. 26:89-100.

Dian, W., Jiang, H., Wu, P. 2005. Evolution and expression analysis of starch synthase III and IV in rice. J. Exp. Bot. 412:623-632.

Flood, R.G., Lagudah, E.S., Halloran, G.M. 1992. Expression of vernalization requirement and spikelet number in synthetic hexaploid wheats and their Triticum tauschii and tetraploid wheat parents. Ann. Bot. 69:213217.

Gao, M., Wanat, J., Stinard, P.S., James, M.G., Myers, A.M. 1998. Characterization of dull1, a maize gene coding for a novel starch synthase. Plant Cell 10:399-412.

Gororo, N.N., Flood, R.G., Eastwood, R.F., Eagles, H.A. 2001. Photoperiod and vernalization responses in Triticum turgidum $\times$ T. tauschii synthetic hexaploid wheats. Ann. Bot. 88:947-952.

Guzmán, C., Caballero, L., Alvarez, J.B. 2009. Variation in Spanish cultivated einkorn wheat (Triticum monococcum L. ssp. monococcum) as determined by morphological traits and waxy proteins. Genet. Resour. Crop. Evol. 56:601-604.

Guzmán, C., Caballero, L., Alvarez, J.B. 2011. Molecular characterization of the Wx-B1 allelic variants identified in cultivated emmer wheat and comparison with those of durum wheat. Mol. Breeding. 28:403-411.

Guo, G., Lv, D., Yan, X., Subburaj, S., Ge, P., Li, X., Hu, Y., Yan, Y. 2012. Proteome characterization of developing grains in bread wheat cultivars (Triticum aestivum L.). BMC Plant Biol. 12:147.

Hogg, A.C., Gause, K., Hofer, P., Martin, J.M., Graybosch, R.A., Hansen, L.E., Giroux, M.J. 2013. Creation of a high-amylose durum wheat through mutagenesis of starch synthase II (SSIIa). J. Cereal Sci. 57:377-383.

Huang, X.Q., Brûlé-Babel, A. 2010. Development of genome-specific primers for homoeologous genes in allopolyploid species: the waxy and starch synthase II genes in allohexaploid wheat (Triticum aestivum L.) as examples. BMC Res. Notes 3:140.

Huang, S., Sirikhachornkit, A., Su, X., Faris, J., Gill, B.S., Haselkorn, B., Gornicki, P. 2002. Genes encoding plastid acetyl-CoA carboxylase and 3-phosphoglycerate kinase of the Triticum/Aegilops complex and the evolutionary history of wheat. Proc. Natl. Acad. Sci. USA 99:8133-8138. 
Hurkman, W.J., McCue, K.F., Altenbach, S.B., Korn, A., Tanaka, C.K., Kothari, K.M., Johnson, E.L., Bechtel, D.B., Wilson, J.D., Anderson, O.D, DuPont, F.M. 2003. Effect of temperature on expression of genes encoding enzymes for starch biosynthesis in developing wheat endosperm. Plant Sci. 164:873-881.

James, M.G., Denyer, K., Myers, A.M. 2003. Starch synthesis in the cereal endosperm. Curr. Opin. Plant. Biol. 6:215-222.

Kang, G.Z., Wang, Y.H., Liu, C., Shen, B.Q., Zheng, B.B., Feng, W., Guo, T.C. 2010. Difference in AGPase subunits could be associated with starch accumulation in grains between two wheat cultivars. Plant Growth Regul. 61:61-66.

Konik-Rose, C.M., Rahman, S., Appels, R., Moss, R., McMaster, G., Marshall, D.R., Stoddard, F.L. 2009. Starch characterisation and variability in GBSS loci of synthetic hexaploid wheats and their durum and Aegilops tauschii parents. Euphytica 167:203-216.

Li, Z., Sun, F., Xu, S., Chu, X., Mukai, Y., Yamamoto, M., Ali, S., Rampling, L., Kosar-Hashemi, B., Rahman, S., Morell, M.K. 2003. The structural organisation of the gene encoding class II starch synthase of wheat and barley and the evolution of the genes encoding starch synthases in plants. Funct. Integr. Genomics 3:76-85.

Li, W., Gao, Z., Xiao, W., Wei, Y.M., Liu, Y.X., Chen, G.Y., Pu, Z.E., Chen, H.P., Zheng, Y.L. 2012. Molecular diversity of restriction enzyme sites, indels and upstream open reading frames (uORFs) of 5' untransalted regions (UTRs) of Waxy genes in Triticum L. and Aegilops L. species. Genet. Resour. Crop Evol. 59:16251647.

Li, W., Fu, B.B., Li, Z., Liu, Y.X., Pu, Z.E., Qi, P.F., Jiang, Q.T., Chen, G.Y., Wang, J.R., Wei, Y.M., Zheng, Y.L. 2016. Characterization of the waxy gene in diploid Triticum L. and Aegilops L. species and its geographic distribution. Genet. Resour. Crop Evol. 63:987-1002.

Limin, A.E., Houde, M., Chauvin, L.P., Fowler, D.B., Sarhan, F. 1995. Expression of the cold-induced wheat gene Wcs120 and its homologs in related species and interspecific combinations. Genome. 38:1023-1031.

Liu, B., Vega J.M., Feldman M. 1998. Rapid genomic changes in newly synthesized amphiploids of Triticum and Aegilops. II. Changes in low-copy coding DNA sequences. Genome 41:535-542.

Ma, H., Singh, R.P., Mujeeb-Kazi, A. 1995. Resistance to stripe rust in Triticum turgidum, T. tauschii and their synthetic hexaploids. Euphytica 82:117-124.

Martin, C., Smith, A.M. 1995. Starch biosynthesis. Plant Cell 7:971-85.

Murai, J., Taira, T., Ohta, D. 1999. Isolation and characterization of the three Waxy genes encoding the granulebound starch synthase in hexaploid wheat. Gene 234:71-79.

Nieto-Taladriz, M.T., Rodríguez-Quijano, M., Carrillo, J.M. 2000. Polymorphism of waxy proteins in Spanish durum wheats. Plant Breed. 119:277-279.

Ozkan, H., Levy, A.A., Feldman, M. 2001. Allopolyploidy-induced rapid genome evolution in the wheat (Aegilops-Triticum) group. Plant Cell 13:1735-1747.

Pena, R.J., Zarco-Hernandez, J., Mujeeb-Kazi, A. 1995. Glutenin subunit compositions and bread-making quality characteristics of synthetic hexaploid wheats derived from Triticum turgidum $\times$ Triticum tauschii (Coss.) Schmal crosses. J. Cereal Sci. 21:15-23.

Pfaffl, M.W. 2001. A new mathematical model for relative quantification in real-time RT-PCR. Nucleic Acids Res. 29:e45-e45.

Satoh, H., Shibahara, K., Tokunaga, T., Nishi, A., Tasaki, M., Hwang, S., Okita, T.W., Kaneko, N., Fujita, N., Yoshida, M., Hosaka, Y., Sato A., Utsumi, Y., Ohdan, T., Nakamura, Y. 2008. Mutation of the plastidial $\alpha$-glucan phosphorylase gene in rice affects the synthesis and structure of starch in the endosperm. Plant Cell. 20(7):1833-1849.

Sestili, F., Botticella, E., Bedo, Z., Phillips, A., Lafiandra, D. 2010. Production of novel allelic variation for genes involved in starch biosynthesis through mutagenesis. Mol. Breeding 25:145-154.

Smith, A.M., Denyer, K., Martin, C.R. 1995. What controls the amount and structure of starch in storage organs?. Plant Physiol. 107:673-677.

Tetlow, I.J., Morell, M.K., Emes, M.J. 2004. Recent developments in understanding the regulation of starch metabolism in higher plants. J. Exp. Bot. 55:2131-2145.

Tomlinson, K., Denyer, K. 2003. Starch synthesis in cereal grains. Adv. Bot. Res. 40:1-61. 
Tickle, P., Burrell, M.M., Coates, S.A., Emes, M., Tetlow, I.J., Bowsher, C.G. 2009. Characterization of plastidial starch phosphorylase in Triticum aestivum L. endosperm. J. Plant Phsiol. 166:1465-1478.

Villareal, R.L., Mujeeb-Kazi, A., Del Toro, E., Crossa, J., Rajaram, S. 1994. Agronomic variability in selected Triticum turgidum $\times$ T. tauschii synthetic hexaploid wheats. J. Agron. Crop Sci. 173:307-317.

Watanabe, N., Noda, Y., Goto, N., Miura, H. 1998. Variation for apparent amylose content of endosperm starch in Triticum durum and Aegilops squarrosa. Euphytica 101:283-286.

Yamamor, M., Guzmán, C. 2013. SNPs and an insertion sequence in five Wx-A1 alleles as factors for variant Wx-A1 protein in wheat. Euphytica 192:325-338.

Zhang, L.Q., Liu, D.C., Yan, Z.H., Lan, X.J., Zheng, Y.L., Zhou, Y.H. 2004. Rapid changes of microsatellite flanking sequence in the allopolyploidization of new synthesized hexaploid wheat. Sci. China C Life Sci. 47:553-561.

Zhang, C., Jiang, D., Liu, F., Cai, J., Dai, T., Cao, W. 2010. Starch granules size distribution in superior and inferior grains of wheat is related to enzyme activities and their gene expressions during grain filling. J. Cereal Sci. 51:226-233.

Zhu, T., Jackson, D.S., Wehling, R.L., Geera, B. 2008. Comparison of amylose determination methods and the development of a dual wavelength iodine binding technique. Cereal Chem. 85:51-58. 